\title{
Weighted Neighbourhood Sequences in Non-Standard Three-Dimensional Grids - Metricity and Algorithms
}

\author{
Robin Strand ${ }^{1}$ and Benedek Nagy ${ }^{2}$ \\ ${ }^{1}$ Centre for Image Analysis, Uppsala University, \\ Box 337, SE-75105 Uppsala, Sweden \\ 2 Department of Computer Science, Faculty of Informatics, University of Debrecen, \\ PO Box 12, 4010, Debrecen, Hungary \\ robin@cb.uu.se, nbenedek@inf.unideb.hu
}

\begin{abstract}
Recently, a distance function was defined on the facecentered cubic and body-centered cubic grids by combining weights and neighbourhood sequences. These distances share many properties with traditional path-based distance functions, such as the city-block distance, but are less rotational dependent. We present conditions for metricity and algorithms to compute the distances.
\end{abstract}

\section{Introduction}

When using non-standard grids such as the face-centered cubic (fcc) grid and the body-centered cubic (bcc) grid for 3D images, less samples are needed to obtain the same representation/reconstruction quality compared to the cubic grid [1]. This is one reason for the increasing interest in using these grids in, e.g., image acquisition [1, image processing [234, and visualization [5].

Measuring distances is of great importance in many applications. Because of its low rotational dependency, the Euclidean distance is often used as distance function. There are, however, applications where other distance functions are better suited. For example, when minimal cost-paths are computed, a distance function defined as the minimal cost-path between any two points is better suited, see, e.g., 7, where the constrained distance transform is computed using the Euclidean distance resulting in a complex algorithm. The corresponding algorithm using a path-based approach is simple, fast, and easy to generalize to higher dimensions 89. Examples of path-based distances are weighted distances, where weights define the cost (distance) between neighbouring grid points $\left[\begin{array}{l|l|l|l|}10 & 3 & 2\end{array}\right.$, and distances based on neighbourhood sequences (NS-distances), where the adjacency relation is allowed to vary along the path, but all steps are of unit weights 114. These path-based distance functions are generalizations of the well-known city-block and chessboard distance function defined for the square grid in 12 .

Neighbourhood sequences was mentioned in [13, where vectors (steps) of various vector sets were periodically used to construct a shortest path between two

D. Coeurjolly et al. (Eds.): DGCI 2008, LNCS 4992, pp. 201212, 2008.

(C) Springer-Verlag Berlin Heidelberg 2008 
points. A general definition of digital distances allowing both weights and neighbourhood sequences was presented in [14. For the case with no weights, they presented non-metrical distances and conditions for the neighbourhood sequence to define metrical distance.

Das and his coathors 15 chosed the natural neighbourhood conditions of $\mathbb{Z}^{n}$ and computed several results on digital distances by neighbourhood sequences but without using any weights. Note that in the square grid one of these so-called octagonal distances is mentioned already in 1968 [11] as a relatively good and simple approximation to the Euclidean distance in a digital way.

Metrical distances are of importance both in theory and in applications. The necessary and sufficient condition for a neighbourhood sequence (without weights) to define a metric distance can be found in [15] for periodic neighbourhood sequences (NS) on $\mathbb{Z}^{n}$, in [16] for the non-periodic case, in [4] for the fcc and bcc grids.

In [17] NS and weights were together used in the sense of [14], but with the well-known natural neighbourhood structure of $\mathbb{Z}^{2}$. In [17], the basic theory for weighted distances based on neighbourhood sequences (weighted NS-distances) is presented including a formula for the distance between two points, conditions for metricity, optimal weight calculation, and an algorithm to compute the distance transform. In [18, some results for weighted NS-distances on the fcc and bcc grids were presented. In this paper, we further develop the theory for weighted NS-distances on the fcc and bcc grids by presenting sufficient conditions for metricity and algorithms that can be used to compute the distance transform and a minimal cost-path between two points.

Note that the results presented here also apply to weighted distances and NSdistances, since they are both special cases of the proposed distance function.

\section{Basic Notions and Previous Results}

The following definitions of the fcc and bcc grids are used:

$$
\begin{aligned}
& \mathbb{F}=\{(x, y, z): x, y, z \in \mathbb{Z} \text { and } x+y+z \equiv 0 \quad(\bmod 2)\} \\
& \mathbb{B}=\{(x, y, z): x, y, z \in \mathbb{Z} \text { and } x \equiv y \equiv z \quad(\bmod 2)\}
\end{aligned}
$$

When the result is valid for both $\mathbb{F}$ and $\mathbb{B}$, the notation $\mathbb{G}$ is used. Two distinct points $\mathbf{p}_{1}=\left(x_{1}, y_{1}, z_{1}\right), \mathbf{p}_{2}=\left(x_{2}, y_{2}, z_{2}\right) \in \mathbb{G}$ are $\rho$-neighbours, $1 \leq \rho \leq 2$, if

1. $\left|x_{1}-x_{2}\right|+\left|y_{1}-y_{2}\right|+\left|z_{1}-z_{2}\right| \leq 3$ and

2. $\max \left\{\left|x_{1}-x_{2}\right|,\left|y_{1}-y_{2}\right|,\left|z_{1}-z_{2}\right|\right\} \leq \rho$

The points $\mathbf{p}_{1}, \mathbf{p}_{2}$ are adjacent if $\mathbf{p}_{1}$ and $\mathbf{p}_{2}$ are $\rho$-neighbours for some $\rho$. The $\rho$-neighbours which are not $(\rho-1)$-neighbours are called strict $\rho$-neighbours. The neighbourhood relations are visualized in Figure 1 by showing the Voronoi regions, i.e. the voxels, corresponding to some adjacent grid points.

A NS $B$ is a sequence $B=(b(i))_{i=1}^{\infty}$, where each $b(i)$ denotes a neighbourhood relation in $\mathbb{G}$. If $B$ is periodic, i.e., if for some fixed strictly positive $l \in \mathbb{Z}_{+}$, 

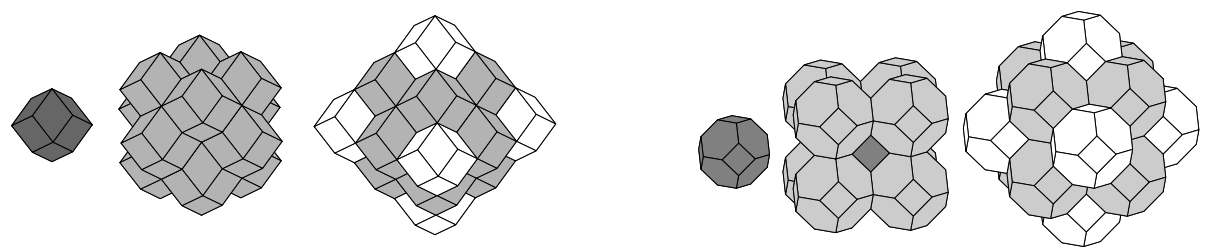

Fig. 1. The grid points corresponding to the dark and the light grey voxels are 1neighbours. The grid points corresponding to the dark grey and white voxels are (strict) 2-neighbours. Left: fcc, right: bcc.

$b(i)=b(i+l)$ is valid for all $i \in \mathbb{Z}_{+}$, then we write $B=(b(1), b(2), \ldots, b(l))$. A path, denoted $\mathcal{P}$, in a grid is a sequence $\mathbf{p}_{0}, \mathbf{p}_{1}, \ldots, \mathbf{p}_{n}$ of adjacent grid points. A path between $\mathbf{p}_{0}$ and $\mathbf{p}_{n}$ is denoted $\mathcal{P}_{\mathbf{p}_{0}, \mathbf{p}_{n}}$. A path is a $B$-path of length $n$ if, for all $i \in\{1,2, \ldots, n\}, \mathbf{p}_{i-1}$ and $\mathbf{p}_{i}$ are $b(i)$-neighbours. The notation 1 - and (strict) 2-steps will be used for a step to a 1-neighbour and step to a (strict) 2 -neighbour, respectively. The number of 1-steps and strict 2 -steps in a given path $\mathcal{P}$ is denoted $\mathbf{1}_{\mathcal{P}}$ and $\mathbf{2}_{\mathcal{P}}$, respectively.

Definition 1. Given the NS B, the NS-distance $d\left(\mathbf{p}_{0}, \mathbf{p}_{n} ; B\right)$ between the points $\mathbf{p}_{0}$ and $\mathbf{p}_{n}$ is the length of (one of) the shortest B-path (s) between the points.

Let the real numbers $\alpha$ and $\beta$ (the weights) and a path $\mathcal{P}$ of length $n$, where exactly $l(l \leq n)$ adjacent grid points in the path are strict 2 -neighbours, be given. The length of the $(\alpha, \beta)$-weighted $B$-path $\mathcal{P}$ is $(n-l) \alpha+l \beta$. The $B$-path $\mathcal{P}$ between the points $\mathbf{p}_{0}$ and $\mathbf{p}_{n}$ is a minimal cost $(\alpha, \beta)$-weighted $B$-path between the points $\mathbf{p}_{0}$ and $\mathbf{p}_{n}$ if no other $(\alpha, \beta)$-weighted $B$-path between the points is shorter than the length of the $(\alpha, \beta)$-weighted $B$-path $\mathcal{P}$.

Definition 2. Given the NSB and the weights $\alpha, \beta$, the weighted NS-distance $d_{\alpha, \beta}\left(\mathbf{p}_{0}, \mathbf{p}_{n} ; B\right)$ is the length of (one of) the minimal cost $(\alpha, \beta)$-weighted $B$ path $(s)$ between the points.

The following notation is used:

$$
\begin{aligned}
& \mathbf{1}_{B}^{k}=|\{i: b(i)=1,1 \leq i \leq k\}| \text { and } \\
& \mathbf{2}_{B}^{k}=|\{i: b(i)=2,1 \leq i \leq k\}| .
\end{aligned}
$$

We now recall from [18, the following two theorems giving the distance between two grid points $(0,0,0)$ and $(x, y, z)$, where $x \geq y \geq z \geq 0$. We remark that by translation-invariance and symmetry, the distance between any two grid points is given by the formula below.

Remark 1. Hereafter, we will refer to "the weights $\alpha$ and $\beta$ " as real numbers $\alpha$ and $\beta$ such that $0<\alpha \leq \beta \leq 2 \alpha$. This is natural since

- a 2-step should be more expensive than a 1-step since strict 2-neighbours are intuitively at a larger distance than 1-neighbours (projection property) and 
- two 1-steps should be more expensive than a 2-step - otherwise no 2-steps would be used in a minimal cost-path.

Theorem 1. Let the $N S B$, the weights $\alpha, \beta$ and the point $(x, y, z) \in \mathbb{F}$, where $x \geq y \geq z \geq 0$, be given. The weighted $N S$-distance between $\mathbf{0}$ and $(x, y, z)$ is given by

$$
\begin{aligned}
d_{\alpha, \beta}(\mathbf{0},(x, y, z) ; B) & =\left\{\begin{array}{cc}
k \cdot \alpha & \text { if } x \leq y+z \\
(2 k-x) \cdot \alpha+(x-k) \cdot \beta & \text { otherwise, }
\end{array}\right. \\
\text { where } k & =\min _{k}: k \geq \max \left(\frac{x+y+z}{2}, x-\mathbf{2}_{B}^{k}\right) .
\end{aligned}
$$

Theorem 2. Let the $N S B$, the weights $\alpha, \beta$, and the point $(x, y, z) \in \mathbb{B}$, where $x \geq y \geq z \geq 0$, be given. The weighted $N S$-distance between $\mathbf{0}$ and $(x, y, z)$ is given by

$$
\begin{aligned}
d_{\alpha, \beta}(\mathbf{0},(x, y, z) ; B) & =(2 k-x) \cdot \alpha+(x-k) \cdot \beta \\
\text { where } k & =\min _{k}: k \geq \max \left(\frac{x+y}{2}, x-\mathbf{2}_{B}^{k}\right) .
\end{aligned}
$$

We collect some previous results about paths generating NS-distances and weighted NS-distances:

Lemma 1. Let the $N S B$, the weights $\alpha, \beta$, and the point $\mathbf{p}=(x, y, z) \in \mathbb{G}$, where $x \geq y \geq z \geq 0$ be given. Then there is a path $\mathcal{P}_{\mathbf{0}, \mathbf{p}}$ such that

i $\mathcal{P}_{\mathbf{0}, \mathbf{p}}$ is a shortest $B$-path,

ii $\mathcal{P}_{\mathbf{0}, \mathbf{p}}$ is a minimal cost $(\alpha, \beta)$-weighted $B$-path, and

iii $\mathcal{P}_{\mathbf{0}, \mathbf{p}}$ consists only of the steps

$$
\begin{array}{r}
(2,0,0),(1,1,0),(1,-1,0),(0,1,1), \text { and }(1,0,1) \quad(f c c) \\
(2,0,0),(1,1,-1),(1,1,1) \text {, and }(1,-1,-1)(b c c)
\end{array}
$$

Proof. i and iii follow from Theorem 2 and Theorem 5 and their proofs in [4 for the fcc and bcc grids, respectively and ii follows from Lemma 4 in [18].

Remark 2. It follows from Lemma 1 that $\mathrm{i}$ and ii are valid for any point $\mathbf{p} \in \mathbb{G}$. Also, for any point $\mathbf{p} \in \mathbb{G}$, the paths can be limited to a few local steps as in iii by changing the sign and permuting the positions of the coordinates of the local steps.

\section{Metricity of the Distance Functions}

Example 1. Let $B=(2,2,1), \alpha=2$, and $\beta=3$. For the points $\mathbf{p}_{1}=(0,0,0)$, $\mathbf{p}_{2}=(4,0,0)$, and $\mathbf{p}_{3}=(6,0,0)$ in fcc or bcc, we have

$$
d_{\alpha, \beta}\left(\mathbf{p}_{1}, \mathbf{p}_{3} ; B\right)=10>9=6+3=d_{\alpha, \beta}\left(\mathbf{p}_{1}, \mathbf{p}_{2} ; B\right)+d_{\alpha, \beta}\left(\mathbf{p}_{2}, \mathbf{p}_{3} ; B\right),
$$

so the triangular inequality is violated and therefore, $d_{2,3}(\cdot, \cdot ;(2,2,1))$ is not a metric on neither $\mathbb{F}$ nor $\mathbb{B}$. 
It is obvious that the distance functions are positive definite for positive weights. They are also symmetric since the fcc and bcc grids are point-lattices. To establish the condition of metricity, only the triangular inequality must be proved. As Example 1 shows, the triangular inequality is related to the weights and the number of occurences of 1 and 2 and their positions in the NS. We keep the weights within the interval in Remark 1 and to prove the main result about metricity, Theorem 3, we need some conditions also on the NS, namely: Given three points $\mathbf{p}_{1}, \mathbf{p}_{2}$, and $\mathbf{p}_{3}$ in $\mathbb{G}$, there are minimal cost-paths $\mathcal{P}_{\mathbf{p}_{1}, \mathbf{p}_{2}}, \mathcal{P}_{\mathbf{p}_{2}, \mathbf{p}_{3}}$, $\mathcal{P}_{\mathbf{p}_{1}, \mathbf{p}_{3}}$ such that

$$
\begin{aligned}
& \mathbf{1}_{\mathcal{P}_{\mathbf{p}_{1}, \mathbf{p}_{3}}}+\mathbf{2}_{\mathcal{P}_{\mathbf{p}_{1}, \mathbf{p}_{3}}} \leq \mathbf{1}_{\mathcal{P}_{\mathbf{p}_{1}, \mathbf{p}_{2}}}+\mathbf{2}_{\mathcal{P}_{\mathbf{p}_{1}, \mathbf{p}_{2}}}+\mathbf{1}_{\mathcal{P}_{\mathbf{p}_{2}, \mathbf{p}_{3}}}+\mathbf{2}_{\mathcal{P}_{\mathbf{p}_{2}, \mathbf{p}_{3}}} \text { and } \\
& \mathbf{1}_{\mathcal{P}_{\mathbf{p}_{1}, \mathbf{p}_{3}}}+2 \cdot \mathbf{2}_{\mathcal{P}_{\mathbf{p}_{1}, \mathbf{p}_{3}}} \leq \mathbf{1}_{\mathcal{P}_{\mathbf{p}_{1}, \mathbf{p}_{2}}}+2 \cdot \mathbf{2}_{\mathcal{P}_{\mathbf{p}_{1}, \mathbf{p}_{2}}}+\mathbf{1}_{\mathcal{P}_{\mathbf{p}_{2}, \mathbf{p}_{3}}}+2 \cdot \mathbf{2}_{\mathcal{P}_{\mathbf{p}_{2}, \mathbf{p}_{3}}} \text {. }
\end{aligned}
$$

Eq. (3) is the triangular inequality for NS-distances and is given by the metricity of NS-distance proved in 4]. Eq. (4) can be interpreted the lengths of the paths obtained by replacing each 2 -step by two 1-steps. Eq. (4) is not fulfilled for all shortest paths, as the following example shows.

Example 2. Consider the fcc grid with $B=(2)$ and $\mathbf{p}_{1}=(0,0,0), \mathbf{p}_{3}=(2,2,0)$. The path $(0,0,0),(2,0,0),(2,2,0)$ is a shortest path, and (3) is fulfilled for any point $\mathbf{p}_{2}$. Eq (4) is not fulfilled with $\mathbf{p}_{2}=(1,1,0)$ since $\mathbf{1}_{\mathcal{P}_{\mathbf{p}_{1}, \mathbf{p}_{3}}}=0, \mathbf{2}_{\mathcal{P}_{\mathbf{p}_{1}, \mathbf{p}_{3}}}=2$, $\mathbf{1}_{\mathcal{P}_{\mathbf{p}_{1}, \mathbf{p}_{2}}}=1, \mathbf{2}_{\mathcal{P}_{\mathbf{p}_{1}, \mathbf{p}_{2}}}=0, \mathbf{1}_{\mathcal{P}_{\mathbf{p}_{2}, \mathbf{p}_{3}}}=1$, and $\mathbf{2}_{\mathcal{P}_{\mathbf{p}_{2}, \mathbf{p}_{3}}}=0$

There are, however, always shortest paths (that are also minimal cost-paths) for any $\mathbf{p}_{1}, \mathbf{p}_{2}$, and $\mathbf{p}_{3}$ such that also (4) is fulfilled. This is proved in Lemma 3 and 5 below. For Example 2, (44) is fulfilled with the path $\mathcal{P}_{\mathbf{p}_{1}, \mathbf{p}_{3}}$ defined by $(0,0,0)$, $(1,1,0),(2,2,0)$ and any $\mathbf{p}_{2}$. We will see that (3) together with $\alpha \leq \beta$ and (4) together with $\beta \leq 2 \alpha$ implies metricity also for the $(\alpha, \beta)$-weighted $B$-distance.

\subsection{The fcc Grid}

Lemma 2. Let the weights $\alpha, \beta$ and the $N S B$ be given. There is a minimal $\operatorname{cost}(\alpha, \beta)$-weighted $B$-path $\mathcal{P}=\mathcal{P}_{\mathbf{p}_{1}, \mathbf{p}_{2}}$ defining the $(\alpha, \beta)$-weighted $N S$-distance between $\mathbf{p}_{1}=\left(x_{1}, y_{1}, z_{1}\right) \in \mathbb{F}$ and $\mathbf{p}_{2}=\left(x_{2}, y_{2}, z_{2}\right) \in \mathbb{F}$ such that

$$
\mathbf{1}_{\mathcal{P}}+2 \cdot \mathbf{2}_{\mathcal{P}} \geq \max \left\{\left|x_{2}-x_{1}\right|,\left|y_{2}-y_{1}\right|,\left|z_{2}-z_{1}\right|\right\}
$$

The equality is attained when

$$
\max \left\{\left|x_{2}-x_{1}\right|,\left|y_{2}-y_{1}\right|,\left|z_{2}-z_{1}\right|\right\} \geq \frac{\left|x_{2}-x_{1}\right|+\left|y_{2}-y_{1}\right|+\left|z_{2}-z_{1}\right|}{2} .
$$

Proof. Given a point $(x, y, z)$ such that $x \geq y \geq z \geq 0$, we use a path $\mathcal{P}^{\prime}$ between $\mathbf{0}$ and $(x, y, z)$ satisfying $\mathrm{i}-$ iii in Lemma 1 By Lemma 2 in [18, we have

$$
\mathbf{1}_{\mathcal{P}^{\prime}}=\left\{\begin{array}{cl}
\hat{k} & \text { if } x \leq y+z \\
2 \hat{k}-x & \text { otherwise. }
\end{array} \text { and } \mathbf{2}_{\mathcal{P}^{\prime}}=\left\{\begin{array}{cl}
0 & \text { if } x \leq y+z \\
x-\hat{k} & \text { otherwise }
\end{array}\right.\right.
$$


where $\hat{k}=\min \left\{k: k \geq \max \left(\frac{x+y+z}{2}, x-\mathbf{2}_{B}^{k}\right)\right\}$.

We recall that for $x \leq y+z, \hat{k}=\frac{x+y+z}{2}$ (see the proof of Theorem 2 in [4]). Thus when $x \leq y+z, \mathbf{1}_{\mathcal{P}^{\prime}}+2 \cdot \mathbf{2}_{\mathcal{P}^{\prime}}=\hat{k}=\frac{x+y+z}{2} \geq x=\max \{x, y, z\}$.

For the case $x \geq \frac{x+y+z}{2}$, i.e. $x \geq y+z, \mathbf{1}_{\mathcal{P}^{\prime}}+2 \cdot \mathbf{2}_{\mathcal{P}^{\prime}}=2 \hat{k}-x+2(x-\hat{k})=x$.

This shows that the lemma holds for $\left(x_{1}, y_{1}, z_{1}\right)=(0,0,0)$ and $\left(x_{2}, y_{2}, z_{2}\right)=$ $(x, y, z)$. The lemma holds also for the general case by translation invariance and symmetry.

Lemma 3. Let the weights $\alpha, \beta$ and the $N S B$ such that

$$
\sum_{i=1}^{N} b(i) \leq \sum_{i=j}^{j+N-1} b(i) \forall j, N \geq 1
$$

be given. For any points $\mathbf{p}_{1}=\left(x_{1}, y_{1}, z_{1}\right), \mathbf{p}_{2}=\left(x_{2}, y_{2}, z_{2}\right), \mathbf{p}_{3}=\left(x_{3}, y_{3}, z_{3}\right) \in \mathbb{F}$, there are minimal cost $(\alpha, \beta)$-weighted $B$-paths $\mathcal{P}_{\mathbf{p}_{1}, \mathbf{p}_{3}}, \mathcal{P}_{\mathbf{p}_{1}, \mathbf{p}_{2}}, \mathcal{P}_{\mathbf{p}_{1}, \mathbf{p}_{3}}$ such that

$$
\mathbf{1}_{\mathcal{P}_{\mathbf{p}_{1}, \mathbf{p}_{3}}}+2 \cdot \mathbf{2}_{\mathcal{P}_{\mathbf{p}_{1}, \mathbf{p}_{3}}} \leq \mathbf{1}_{\mathcal{P}_{\mathbf{p}_{1}, \mathbf{p}_{2}}}+2 \cdot \mathbf{2}_{\mathcal{P}_{\mathbf{p}_{1}, \mathbf{p}_{2}}}+\mathbf{1}_{\mathcal{P}_{\mathbf{p}_{2}, \mathbf{p}_{3}}}+2 \cdot \mathbf{2}_{\mathcal{P}_{\mathbf{p}_{2}, \mathbf{p}_{3}}} .
$$

Proof. Let $\mathbf{p}_{1}$ and $\mathbf{p}_{3}$ be such that $\left|x_{3}-x_{1}\right| \geq\left|y_{3}-y_{1}\right| \geq\left|z_{3}-z_{1}\right|$. We consider two cases:

$$
\begin{aligned}
& -\left|x_{3}-x_{1}\right|>\left|y_{3}-y_{1}\right|+\left|z_{3}-z_{1}\right| \\
& \mathbf{1}_{\mathcal{P}_{\mathbf{p}_{1}, \mathbf{p}_{3}}}+2 \cdot \mathbf{2}_{\mathcal{P}_{\mathbf{p}_{1}, \mathbf{p}_{3}}}=\left|x_{3}-x_{1}\right| \leq\left|x_{2}-x_{1}\right|+\left|x_{3}-x_{2}\right| \leq \mathbf{1}_{\mathcal{P}_{\mathbf{p}_{1}, \mathbf{p}_{2}}}+2 \text {. }
\end{aligned}
$$

$$
-\left|x_{3}-x_{1}\right| \leq\left|y_{3}-y_{1}\right|+\left|z_{3}-z_{1}\right|
$$

$\mathbf{1}_{\mathcal{P}_{\mathbf{p}_{1}, \mathbf{p}_{3}}}+2 \cdot \mathbf{2}_{\mathcal{P}_{\mathbf{p}_{1}, \mathbf{p}_{3}}}=\mathbf{1}_{\mathcal{P}_{\mathbf{p}_{1}, \mathbf{p}_{3}}}+\mathbf{2}_{\mathcal{P}_{\mathbf{p}_{1}, \mathbf{p}_{3}}} \leq \mathbf{1}_{\mathcal{P}_{\mathbf{p}_{1}, \mathbf{p}_{2}}}+\mathbf{2}_{\mathcal{P}_{\mathbf{p}_{1}, \mathbf{p}_{2}}}+\mathbf{1}_{\mathcal{P}_{\mathbf{p}_{2}, \mathbf{p}_{3}}}+$ $\mathbf{2}_{\mathcal{P}_{\mathbf{p}_{2}, \mathbf{p}_{3}}} \leq \mathbf{1}_{\mathcal{P}_{\mathbf{p}_{1}, \mathbf{p}_{2}}}+2 \cdot \mathbf{2}_{\mathcal{P}_{\mathbf{p}_{1}, \mathbf{p}_{2}}}+\mathbf{1}_{\mathcal{P}_{\mathbf{p}_{2}, \mathbf{p}_{3}}}+2 \cdot \mathbf{2}_{\mathcal{P}_{\mathbf{p}_{2}, \mathbf{p}_{3}}}$. Here we used that $\mathbf{2}_{\mathcal{P}_{\mathbf{p}_{1}, \mathbf{p}_{3}}}=0$ by Lemma 2 in [18], the triangular inequality for NS-distances from [4] $\left(d_{(1,1)}\left(\mathbf{p}_{1}, \mathbf{p}_{3}\right)=\mathbf{1}_{\mathcal{P}_{\mathbf{p}_{1}, \mathbf{p}_{3}}}+\mathbf{2}_{\mathcal{P}_{\mathbf{p}_{1}, \mathbf{p}_{3}}}\right.$ by Lemma 1), and that

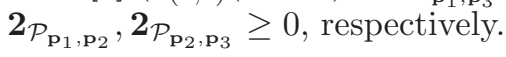

\subsection{The bcc Grid}

Lemma 4. Let the weights $\alpha, \beta$ and the $N S B$ be given. There is a path $\mathcal{P}$ defining the $(\alpha, \beta)$-weighted $N S$-distance between $\left(x_{1}, y_{1}, z_{1}\right) \in \mathbb{B}$ and $\left(x_{2}, y_{2}, z_{2}\right) \in \mathbb{B}$ such that

$$
\mathbf{1}_{\mathcal{P}}+2 \cdot \mathbf{2}_{\mathcal{P}}=\max \left\{\left|x_{2}-x_{1}\right|,\left|y_{2}-y_{1}\right|,\left|z_{2}-z_{1}\right|\right\}
$$

Proof. Given a point $(x, y, z)$ such that $x \geq y \geq z \geq 0$, we use a path $\mathcal{P}^{\prime}$ between 0 and $(x, y, z)$ satisfying $\mathrm{i}-$ iii in Lemma 1]. By Lemma 4 in [18, $\mathcal{P}^{\prime}$ is such that $\mathbf{1}_{\mathcal{P}^{\prime}}=2 \hat{k}-x$ and $\mathbf{2}_{\mathcal{P}^{\prime}}=x-\hat{k}$. Therefore, $\mathbf{1}_{\mathcal{P}^{\prime}}+2 \cdot \mathbf{2}_{\mathcal{P}^{\prime}}=x=\max \{x, y, z\}$. The general case follows by translation invariance and symmetry. 
Lemma 5. Let the weights $\alpha, \beta$ and the NS B be given. For any three points $\mathbf{p}_{1}=\left(x_{1}, y_{1}, z_{1}\right), \mathbf{p}_{2}=\left(x_{2}, y_{2}, z_{2}\right), \mathbf{p}_{3}=\left(x_{3}, y_{3}, z_{3}\right) \in \mathbb{B}$, there are minimal cost $(\alpha, \beta)$-weighted $B$-paths $\mathcal{P}_{\mathbf{p}_{1}, \mathbf{p}_{3}}, \mathcal{P}_{\mathbf{p}_{1}, \mathbf{p}_{2}}, \mathcal{P}_{\mathbf{p}_{1}, \mathbf{p}_{3}}$ such that

$$
\mathbf{1}_{\mathcal{P}_{\mathbf{p}_{1}, \mathbf{p}_{3}}}+2 \cdot \mathbf{2}_{\mathcal{P}_{\mathbf{p}_{1}, \mathbf{p}_{3}}} \leq \mathbf{1}_{\mathcal{P}_{\mathbf{p}_{1}, \mathbf{p}_{2}}}+2 \cdot \mathbf{2}_{\mathcal{P}_{\mathbf{p}_{1}, \mathbf{p}_{2}}}+\mathbf{1}_{\mathcal{P}_{\mathbf{p}_{2}, \mathbf{p}_{3}}}+2 \cdot \mathbf{2}_{\mathcal{P}_{\mathbf{p}_{2}, \mathbf{p}_{3}}} .
$$

Proof. Let $\mathbf{p}_{1}$ and $\mathbf{p}_{3}$ be such that $\left|x_{3}-x_{1}\right| \geq\left|y_{3}-y_{1}\right| \geq\left|z_{3}-z_{1}\right|$. Then $\mathbf{1}_{\mathcal{P}_{\mathbf{p}_{1}, \mathbf{p}_{3}}}+2 \cdot \mathbf{2}_{\mathcal{P}_{\mathbf{p}_{1}, \mathbf{p}_{3}}}=\left|x_{3}-x_{1}\right| \leq\left|x_{2}-x_{1}\right|+\left|x_{3}-x_{2}\right| \leq \mathbf{1}_{\mathcal{P}_{\mathbf{p}_{1}, \mathbf{p}_{2}}}+2 \cdot \mathbf{2}_{\mathcal{P}_{\mathbf{p}_{1}, \mathbf{p}_{2}}}+$ $\mathbf{1}_{\mathcal{P}_{\mathbf{p}_{2}, \mathbf{p}_{3}}}+2 \cdot \mathbf{2}_{\mathcal{P}_{\mathbf{p}_{2}, \mathbf{p}_{3}}}$. Here we used (6) in Lemma 4 with equality, the triangular inequality for real numbers, and (6) in Lemma 4 (again), respectively.

\subsection{Metricity}

Theorem 3. If

$$
\begin{gathered}
\sum_{i=1}^{N} b(i) \leq \sum_{i=j}^{j+N-1} b(i) \forall j, N \geq 1 \text { and } \\
0<\alpha \leq \beta \leq 2 \alpha
\end{gathered}
$$

then $d_{\alpha, \beta}(\cdot, \cdot ; B)$ is a metric on the fcc and bec grids.

Proof. The positive definiteness and symmetry are trivial. We prove the triangular inequality. Let $\mathbf{p}_{1}, \mathbf{p}_{2}, \mathbf{p}_{3} \in \mathbb{G}$ be given. We will prove $d_{(\alpha, \beta)}\left(\mathbf{p}_{1}, \mathbf{p}_{3} ; B\right) \leq$ $d_{(\alpha, \beta)}\left(\mathbf{p}_{1}, \mathbf{p}_{2} ; B\right)+d_{(\alpha, \beta)}\left(\mathbf{p}_{2}, \mathbf{p}_{3} ; B\right)$, i.e. that

$$
\mathbf{1}_{\mathcal{P}_{\mathbf{p}_{1}, \mathbf{p}_{3}}} \alpha+\mathbf{2}_{\mathcal{P}_{\mathbf{p}_{1}, \mathbf{p}_{3}}} \beta \leq \mathbf{1}_{\mathcal{P}_{\mathbf{p}_{1}, \mathbf{p}_{2}}} \alpha+\mathbf{2}_{\mathcal{P}_{\mathbf{p}_{1}, \mathbf{p}_{2}}} \beta+\mathbf{1}_{\mathcal{P}_{\mathbf{p}_{2}, \mathbf{p}_{3}}} \alpha+\mathbf{2}_{\mathcal{P}_{\mathbf{p}_{2}, \mathbf{p}_{3}}} \beta .
$$

In [4, it is proved that when (7) is fulfilled, then the NS-distance defined by $B$ is metric. Therefore, $d_{(1,1)}\left(\mathbf{p}_{1}, \mathbf{p}_{3} ; B\right) \leq d_{(1,1)}\left(\mathbf{p}_{1}, \mathbf{p}_{2} ; B\right)+d_{(1,1)}\left(\mathbf{p}_{2}, \mathbf{p}_{3} ; B\right)$. Thus,

$$
\mathbf{1}_{\mathcal{P}_{\mathbf{p}_{1}, \mathbf{p}_{3}}}+\mathbf{2}_{\mathcal{P}_{\mathbf{p}_{1}, \mathbf{p}_{3}}} \leq \mathbf{1}_{\mathcal{P}_{\mathbf{p}_{1}, \mathbf{p}_{2}}}+\mathbf{2}_{\mathcal{P}_{\mathbf{p}_{1}, \mathbf{p}_{2}}}+\mathbf{1}_{\mathcal{P}_{\mathbf{p}_{2}, \mathbf{p}_{3}}}+\mathbf{2}_{\mathcal{P}_{\mathbf{p}_{2}, \mathbf{p}_{3}}}
$$

for any shortest $B$-paths $\mathcal{P}_{\mathbf{p}_{1}, \mathbf{p}_{3}}, \mathcal{P}_{\mathbf{p}_{1}, \mathbf{p}_{2}}$, and $\mathcal{P}_{\mathbf{p}_{2}, \mathbf{p}_{3}}$. By Lemma 3 and [5, it follows that

$$
\mathbf{1}_{\mathcal{P}_{\mathbf{p}_{1}, \mathbf{p}_{3}}^{\prime}}+2 \cdot \mathbf{2}_{\mathcal{P}_{\mathbf{p}_{1}, \mathbf{p}_{3}}^{\prime}} \leq \mathbf{1}_{\mathcal{P}_{\mathbf{p}_{1}, \mathbf{p}_{2}}^{\prime}}^{\prime}+2 \cdot \mathbf{2}_{\mathcal{P}_{\mathbf{p}_{1}, \mathbf{p}_{2}}^{\prime}}+\mathbf{1}_{\mathcal{P}_{\mathbf{p}_{2}, \mathbf{p}_{3}}^{\prime}}+2 \cdot \mathbf{2}_{\mathcal{P}_{\mathbf{p}_{2}, \mathbf{p}_{3}}^{\prime}} .
$$

for some shortest $B$-paths $\mathcal{P}_{\mathbf{p}_{1}, \mathbf{p}_{3}}^{\prime}, \mathcal{P}_{\mathbf{p}_{1}, \mathbf{p}_{2}}^{\prime}$, and $\mathcal{P}_{\mathbf{p}_{2}, \mathbf{p}_{3}}^{\prime}$.

By Lemma 1, there are paths such that $\mathcal{P}_{\mathbf{p}_{1}, \mathbf{p}_{3}}=\mathcal{P}_{\mathbf{p}_{1}, \mathbf{p}_{3}}^{\prime}, \mathcal{P}_{\mathbf{p}_{1}, \mathbf{p}_{2}}=\mathcal{P}_{\mathbf{p}_{1}, \mathbf{p}_{2}}^{\prime}$, and $\mathcal{P}_{\mathbf{p}_{2}, \mathbf{p}_{3}}=\mathcal{P}_{\mathbf{p}_{2}, \mathbf{p}_{3}}^{\prime}$.

We consider three cases:

i $\mathbf{1}_{\mathcal{P}_{\mathbf{p}_{1}, \mathbf{p}_{3}}}>\mathbf{1}_{\mathcal{P}_{\mathbf{p}_{1}, \mathbf{p}_{2}}}+\mathbf{1}_{\mathcal{P}_{\mathbf{p}_{2}, \mathbf{p}_{3}}}$

By (10) and since $\frac{\alpha}{\beta} \leq 1$ by (8),

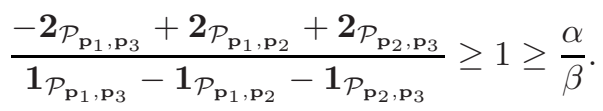

Rewriting this gives (9). 
ii $\mathbf{1}_{\mathcal{P}_{\mathbf{p}_{1}, \mathbf{p}_{3}}}=\mathbf{1}_{\mathcal{P}_{\mathbf{p}_{1}, \mathbf{p}_{2}}}+\mathbf{1}_{\mathcal{P}_{\mathbf{p}_{2}, \mathbf{p}_{3}}}$

By (10) and since $\beta>0$ by (8), (9) is fulfilled.

iii $\mathbf{1}_{\mathcal{P}_{\mathbf{p}_{1}, \mathbf{p}_{3}}}<\mathbf{1}_{\mathcal{P}_{\mathbf{p}_{1}, \mathbf{p}_{2}}}+\mathbf{1}_{\mathcal{P}_{\mathbf{p}_{2}, \mathbf{p}_{3}}}$

By (11) and since $\frac{2 \alpha}{\beta} \geq 1$ by (8),

$$
\frac{-2 \cdot \mathbf{2}_{\mathcal{P}_{\mathbf{p}_{1}, \mathbf{p}_{3}}}+2 \cdot \mathbf{2}_{\mathcal{P}_{\mathbf{p}_{1}, \mathbf{p}_{2}}}+2 \cdot \mathbf{2}_{\mathcal{P}_{\mathbf{p}_{2}, \mathbf{p}_{3}}}}{\mathbf{1}_{\mathcal{P}_{\mathbf{p}_{1}, \mathbf{p}_{3}}}-\mathbf{1}_{\mathcal{P}_{\mathbf{p}_{1}, \mathbf{p}_{2}}}-\mathbf{1}_{\mathcal{P}_{\mathbf{p}_{2}, \mathbf{p}_{3}}}} \leq 1 \leq \frac{2 \alpha}{\beta} .
$$

Rewriting this gives (9).

Thus, (9) holds for all possible cases, so $d_{(\alpha, \beta)}\left(\mathbf{p}_{1}, \mathbf{p}_{3} ; B\right) \leq d_{(\alpha, \beta)}\left(\mathbf{p}_{1}, \mathbf{p}_{2} ; B\right)+$ $d_{(\alpha, \beta)}\left(\mathbf{p}_{2}, \mathbf{p}_{3} ; B\right)$.

Note that using the natural conditions for the weights $\alpha$ and $\beta$, the condition of metricity is exactly the same as for the non-weighted case (see [4]), but the proof is more sophisticated. Thus, to check that a weighted NS distance function is a metric can be done with the same efficiency as for the non-weighted case.

\section{Algorithms}

In [19, weights and neighbourhood sequences that are well-suited for applications are calculated. By minimizing a number of different error-functions measuring the deviation from the Euclidean distance, "optimal" parameters are obtained. Since we are working in digital grids, using the Euclidean distance leads to some unpleasant properties. For instance, the spheres (points with equal distance value from a given point) by the Euclidean distance are not connected (in any sense), opposite to the spheres defined by NS-distances. The Euclidean distance is not integer-valued, which is often preferred in picture analysis; NS-distances are integer-valued and using weights $\alpha, \beta \in \mathbb{N}$ the weighted NS-distance is also integer-valued. Opposite to the weighted distances, the Euclidean distance is not regular on digital grids in the sense of [20]. As mentioned in the introduction, the weighted NS-distance is better suited for the computation of the constrained distance transform. Here, we give algorithms for the constrained distance transform and for finding one minimal cost $(\alpha, \beta)$-weighted $B$-path between any two points.

\subsection{Algorithm to Find a Minimal Cost-Path between Two Points}

The following algorithms construct a shortest weighted NS path from $\mathbf{0}=$ $(0,0,0)$ to the point $\mathbf{p}=(x, y, z)$ with $x \geq y \geq z \geq 0$ in the bcc and fcc grids, respectively. The result is the point sequence $\mathbf{0}=\mathbf{p}_{0}, \mathbf{p}_{1}, \ldots, \mathbf{p}_{m}=\mathbf{q}$ as (one of) the $(\alpha, \beta)$-weighted minimal cost $B$-paths and the distance $d$ between $\mathbf{0}$ and $\mathbf{q}$. The obtained paths satisfy Lemma 1 
Algorithm 1. Constructing a minimal cost $(\alpha, \beta)$-weighted $B$-path on $\mathbb{F}$ Input: NS $B$ and weights $\alpha, \beta$, and a grid point $\mathbf{q} \in \mathbb{F}$.

Output: A minimal cost $(\alpha, \beta)$-weighted $B$-path $\mathcal{P}_{\mathbf{0}, \mathbf{q}}$ and $d=d_{\alpha, \beta}(\mathbf{0}, \mathbf{q} ; B)$. Initialisation: Set $\mathbf{p}_{0} \leftarrow \mathbf{0}, d \leftarrow 0, i \leftarrow 0$.

while $\mathbf{p}_{i} \neq \mathbf{q}$

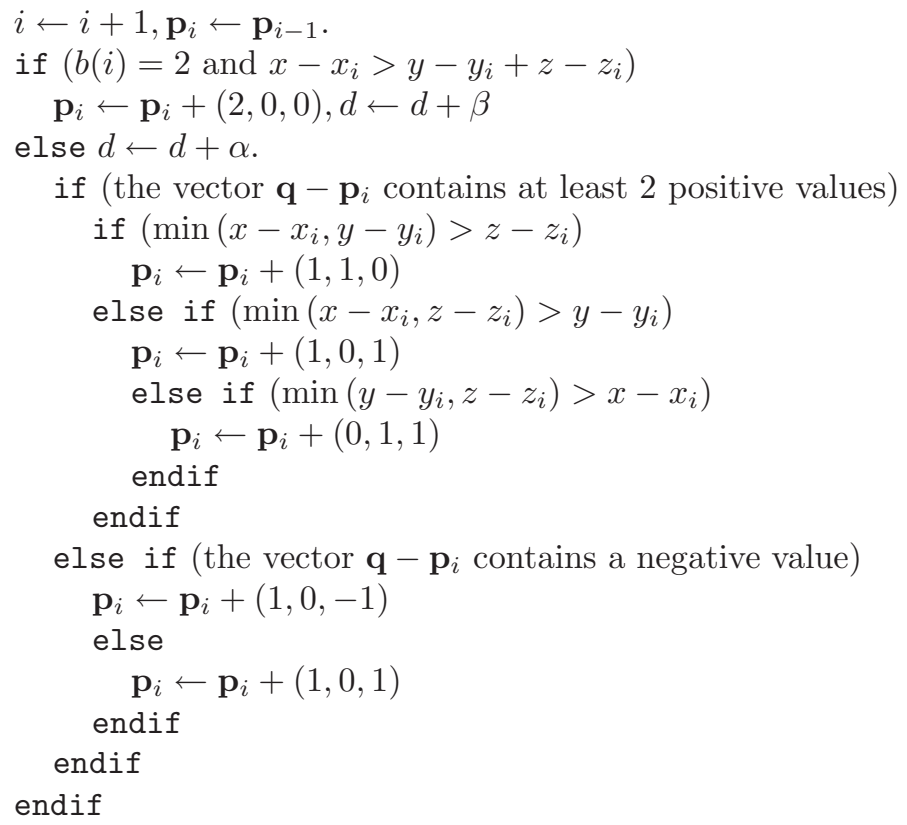

Algorithm 2. Constructing a minimal cost $(\alpha, \beta)$-weighted $B$-path on $\mathbb{B}$ Input: NS $B$ and weights $\alpha, \beta$, and a grid point $\mathbf{q} \in \mathbb{B}$.

Output: A minimal cost $(\alpha, \beta)$-weighted $B$-path $\mathcal{P}_{\mathbf{0}, \mathbf{q}}$ and $d=d_{\alpha, \beta}(\mathbf{0}, \mathbf{q} ; B)$. Initialisation: Set $\mathbf{p}_{0} \leftarrow \mathbf{0}, d \leftarrow 0, i \leftarrow 0$.

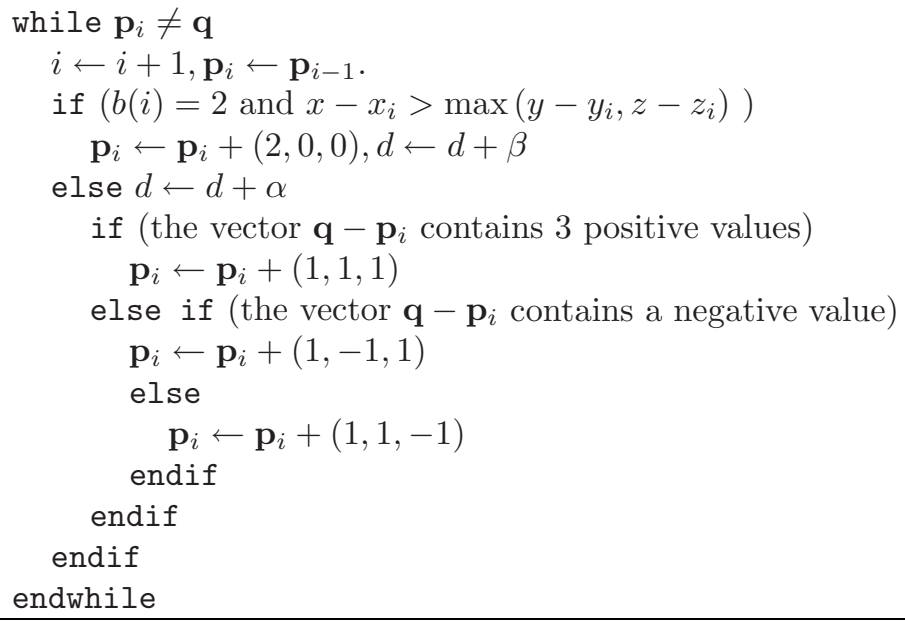




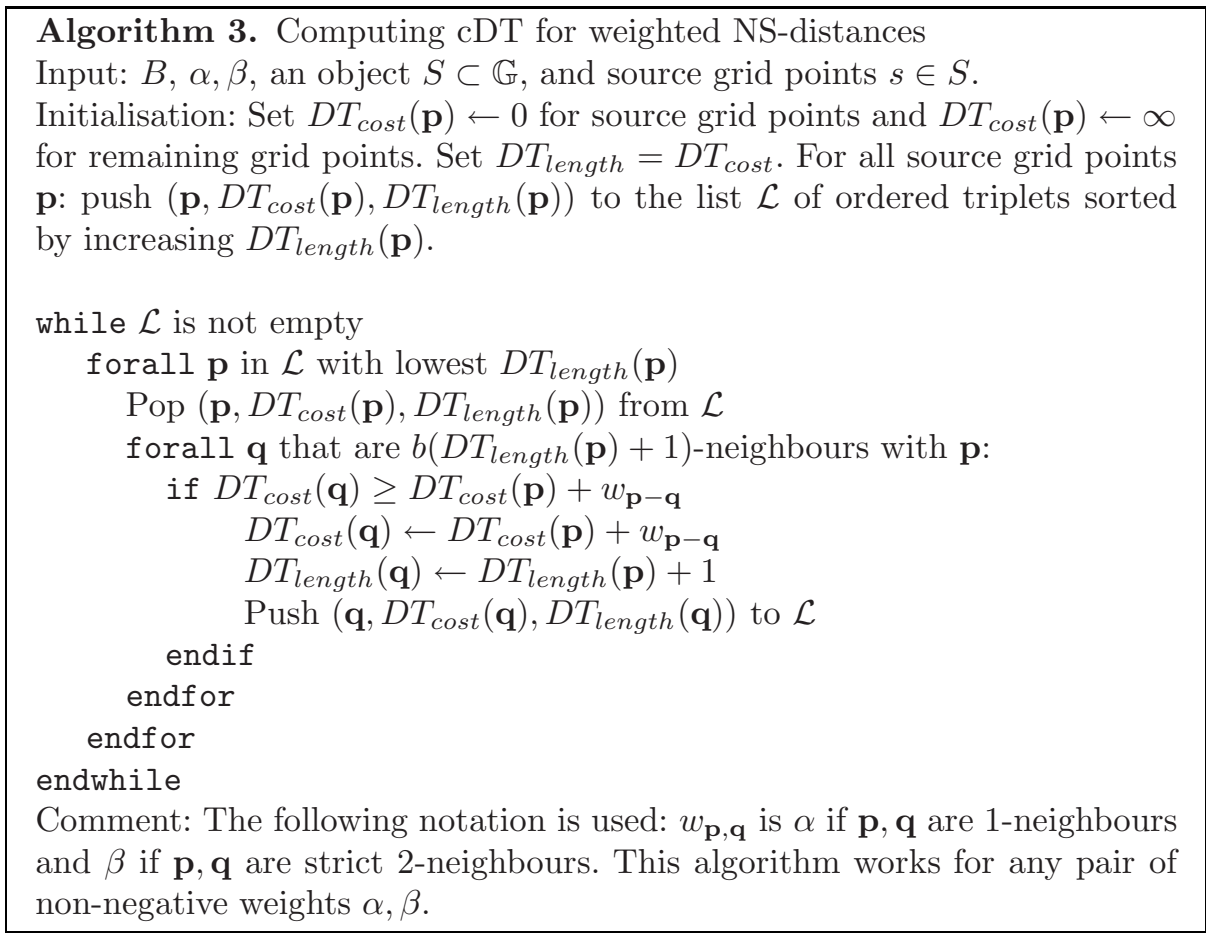

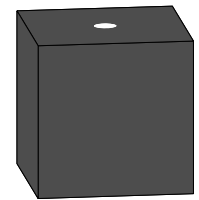

(a)

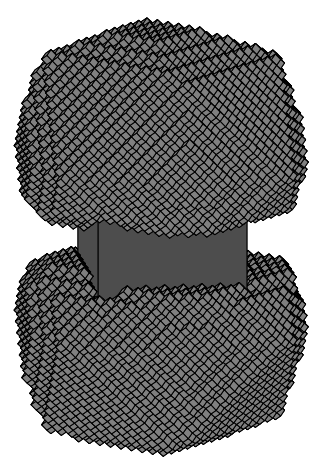

(b)

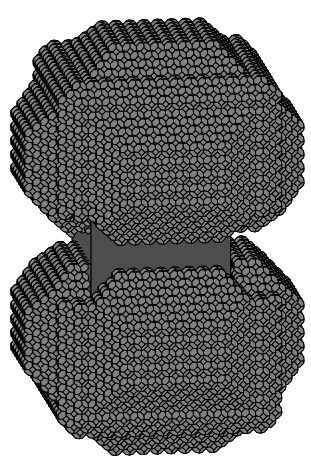

(c)

Fig. 2. The constrained distance transform on the fcc and bcc grids. Figure (a) shows the shape of the obstacle - a cube with a single through-hole. Inside the cube, there is a source grid point. The grid points with a constrained distance less than a threshold $T$ is shown in (b) and (c) for the fcc and bcc grid, respectively. For the fcc grid, $T=38$, $B=(1,2), \alpha=2$, and $\beta=3$ were used and for the bcc grid, $T=110, B=(1,2)$, $\alpha=5$, and $\beta=6$ were used. 


\subsection{Algorithm to Compute the Constrained Distance Transform}

In Algorithm 3 below, pseudo-code for computing the cDT for weighted NSdistances is presented. The basic idea is to update grid points that are neighbours with a grid point in the wave-front. This algorithm is presented in [17] for the square grid and similar algorithms are presented in, e.g., 821 . When $\alpha \neq \beta$, the algorithm, in addition to the distance (the cost), must keep track of the length of the shortest path to the source grid points. The length is needed to determine which neighbourhood that is allowed in each step. Note that only the object grid points $S$, and not the set of obstacle grid points, are processed by the algorithm.

\section{Conclusions}

We have presented sufficient conditions on the weights and neighbourhood sequence for the weighted NS-distance on the fcc and bcc grids to be a metric. The conditions derived here are very natural, for the neighbourhood sequence it is the same condition as metricity for NS-distances (i.e. with unit weights) introduced in [16]. Note that the conditions for metricity on the fcc and bcc grids presented here are the same as for metricity on the square grid, see [17.

The algorithms presented in this paper give different results - Algorithm 1 and 2 compute a minimal cost-path between two points and Algorithm 3 computes the constrained distance transform. The latter have several applications, for example finding the shortest path, see [97] and can also be used for shape representation using centres of maximal balls, see e.g. [22].

\section{Acknowledgements}

The research is supported by Digital geometry with applications in image processing in three dimensions (DIGAVIP) at Center for Image Analysis, Uppsala University.

\section{References}

1. Matej, S., Lewitt, R.M.: Efficient 3D grids for image reconstruction using spherically-symmetric volume elements. IEEE Transactions on Nuclear Science 42(4), 1361-1370 (1995)

2. Strand, R., Borgefors, G.: Distance transforms for three-dimensional grids with non-cubic voxels. Computer Vision and Image Understanding 100(3), 294-311 (2005)

3. Fouard, C., Strand, R., Borgefors, G.: Weighted distance transforms generalized to modules and their computation on point lattices. Pattern Recognition 40(9), 2453-2474 (2007)

4. Strand, R., Nagy, B.: Distances based on neighbourhood sequences in non-standard three-dimensional grids. Discrete Applied Mathematics 155(4), 548-557 (2007) 
5. Carr, H., Theussl, T., Möller, T.: Isosurfaces on optimal regular samples. In: Bonneau, G.-P., Hahmann, S., C.D.H. (eds.) Proceedings of the symposium on Data visualisation 2003, Eurographics Association, pp. 39-48 (2003)

6. Strand, R., Stelldinger, P.: Topology preserving marching cubes-like algorithms on the face-centered cubic grid. In: Proceedings of 14th International Conference on Image Analysis and Processing (ICIAP 2007), Modena, Italy, pp. 781-788 (2007)

7. Coeurjolly, D., Miguet, S., Tougne, L.: 2D and 3D visibility in discrete geometry: an application to discrete geodesic paths. Pattern Recognition Letters 25(5), 561-570 (2004)

8. Verwer, B.J.H., Verbeek, P.W., Dekker, S.T.: An efficient uniform cost algorithm applied to distance transforms. IEEE Transactions on Pattern Analysis and Machine Intelligence 11(4), 425-429 (1989)

9. Strand, R., Malmberg, F., Svensson, S.: Minimal cost-path for path-based distances. In: Petrou, M., Saramäki, T., Erçil, A., Loncaric, S. (eds.) ISPA 2007, pp. 379-384 (2007)

10. Borgefors, G.: Distance transformations in digital images. Computer Vision, Graphics, and Image Processing 34, 344-371 (1986)

11. Rosenfeld, A., Pfaltz, J.L.: Distance functions on digital pictures. Pattern Recognition 1, 33-61 (1968)

12. Rosenfeld, A., Pfaltz, J.L.: Sequential operations in digital picture processing. Journal of the ACM 13(4), 471-494 (1966)

13. Yamashita, M., Honda, N.: Distance functions defined by variable neighborhood sequences. Pattern Recognition 17(5), 509-513 (1984)

14. Yamashita, M., Ibaraki, T.: Distances defined by neighborhood sequences. Pattern Recognition 19(3), 237-246 (1986)

15. Das, P.P., Chakrabarti, P.P.: Distance functions in digital geometry. Information Sciences 42, 113-136 (1987)

16. Nagy, B.: Distance functions based on neighbourhood sequences. Publicationes Mathematicae Debrecen 63(3), 483-493 (2003)

17. Strand, R.: Weighted distances based on neighbourhood sequences. Pattern Recognition Letters 28(15), 2029-2036 (2007)

18. Strand, R.: Weighted distances based on neighbourhood sequences in non-standard three-dimensional grids. In: Ersbøll, B.K., Pedersen, K.S. (eds.) SCIA 2007. LNCS, vol. 4522, pp. 452-461. Springer, Heidelberg (2007)

19. Strand, R., Nagy, B.: Weighted neighbourhood sequences in non-standard threedimensional grids - parameter optimization. In: Brimkov, V.E., Barneva, R.P., Hauptman, H. (eds.) IWCIA 2008. LNCS, vol. 4958, pp. 51-62. Springer, Heidelberg (2008)

20. Klette, R., Rosenfeld, A.: Digital geometry: Geometric methods for digital image analysis. The Morgan Kaufmann Series in Computer Graphics. Morgan Kaufmann, San Francisco (2004)

21. Piper, J., Granum, E.: Computing distance transformations in convex and nonconvex domains. Pattern Recognition 20(6), 599-615 (1987)

22. Strand, R.: Shape representation with maximal path-points for path-based distances. In: Petrou, M., Saramäki, T., Erçil, A., Loncaric, S. (eds.) ISPA 2007, pp. 397-402 (2007) 